\title{
Sister Mary Joseph Nodule and peritoneal carcinomatosis from squamous cell cervical carcinoma
}

\author{
Leila Cristina Soares, ${ }^{1}$ Juliana Procopio Almeida ${ }^{1}$
}

\begin{abstract}
Keywords: Sister Mary Joseph nodule, umbilical metastasis, squamous cervical cancer, peritoneal carcinomatosis
\end{abstract}

\begin{abstract}
Sister Mary Joseph nodule is an eponym assigned to a nurse, first reported in 1949, denoting a rare form of cutaneous umbilical metastasis. The primary neoplasm is usually adenocarcinoma and the gastrointestinal tract is the most common primary site. The present case report describes a 79 year old woman with squamous cell cervical carcinoma. Her disease was managed with radiation and concurrent chemotherapy and no evidence of loco-regional recurrent disease was noted on follow-up examination. Eight months after treatment, gynecological examination suggested recurrent disease. Three weeks later, the patient presented with a painless umbilical nodule and the biopsy revealed a poorly differentiated carcinoma. Computed tomography showed ascites, diffuse peritoneal carcinomatosis including an umbilical nodule, multiple pulmonary nodules, bilateral pleural effusion, and retroperitoneal lymphadenopathy. She died 63 days after this manifestation. Review of the literature shows that a Sister Mary Joseph nodule is a sign of advanced neoplastic disease and is associated with poor prognosis. The treatment remains palliative either by radiation, chemotherapy or surgery.

${ }^{1}$ Rio de Janeiro State University, Rio de Janeiro, Brazil
\end{abstract}

\section{Introduction}

The term "Sister Mary Joseph nodule" (SMJN) has been used since 1949 to denote umbilical metastases. Sister Mary Joseph was a nurse and the first observer to establish a correlation between umbilical nodules and carcinoma. SMJN is a rare but typical cutaneous periumbilical metastasis of internal malignancies, and its recognition is important because it may be the first presenting sign of unknown malignant disease in a patient. ${ }^{1}$

The exact mechanism underlying metastases to the umbilicus remains unknown. Proposed routes for the spread of cancer to the umbilicus include direct extension from the peritoneum and spread via arteries, veins, or lymphatic channels. ${ }^{2}$

SMJN is most commonly found in association with adenocarcinomas, and the most common primary site is the gastrointestinal tract (35$65 \%$ ). This metastasis impairs the quality of life and shortens survival. ${ }^{3}$

Although squamous cervical cancer

Please cite this paper as: Soares LC, Almeida JP Sister Mary Joseph Nodule and peritoneal carcinomatosis from squamous cell cervical carcinoma. Proc Obstet Gynecol. 2013;3(3): Article 5 [ 6 p.]. Available from: http://ir.uiowa.edu/pog/. Free full text article.

Corresponding author: Lelila Cristina Soares, Rio de Janeiro State University, 78 ap 104 Rua Paulino Fernandes, Rio de Janeiro, Brazil, 22270-050 Telephone 5521 88564796. Ics1507@yahoo.com.br

Copyright: (c) 2013 Soares, Almeida. This is an open-access article distributed under the terms of the Creative Commons Attribution License, which permits unrestricted use, distribution, and reproduction in any medium, provided the original author and source are credited. 
is a common occurrence, the association of this condition with skin metastasis is rare.

A search of MEDLINE using search terms "umbilical metastasis* AND squamous cell cervix cancer" yielded 6 cases reported in the literature, none of them associated with peritoneal carcinomatosis.

\section{Case Report}

A 79-year-old Caucasian woman presented with postmenopausal intermittent vaginal bleeding and a vegetative mass external to the cervix. Gynecological examination revealed left parametrium infiltration.

Ultrasonography showed the presence of a $30-\mathrm{mm}$ thickened endometrium, and cervical and endometrial biopsy revealed a poorly differentiated tumor.

Immunohistochemistry showed that the cells were positive for p63 and Pan-Keratin and negative for cytokeratin $5 / 6$ and S100. These features indicated the epithelial origin of the neoplastic cells and, as there were no criteria for squamous cell endometrial carcinoma, the patient was classified as having stage IIB cervical cancer, and the disease was managed with concurrent cisplatin-based chemoradiation. The pelvis was treated with $10 \mathrm{MV}$ photons, $45 \mathrm{~Gy}$ in 25 daily fractions followed by brachytherapy. Follow-up examinations included Papanicolaou smear and pelvic examination for 6 months, which revealed no evidence of loco-regional residual disease.

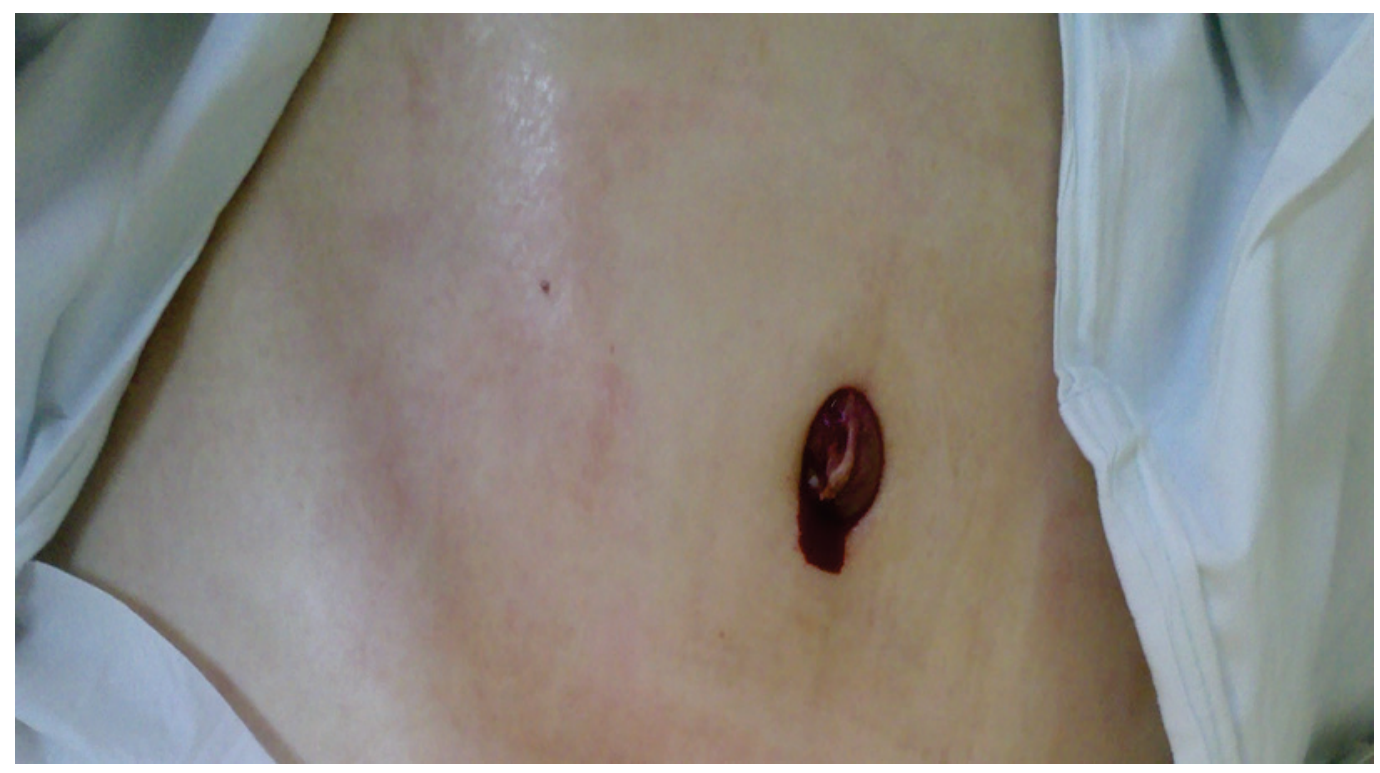

\section{Figure 1: Umbilical nodule}

Eight months after chemoradiotherapy, gynecological examination revealed a pelvic mass and left parametrium infiltration, suggesting recurrent disease. Ultrasonography and magnetic resonance imaging were requested.

Two weeks after this examination, the patient was evaluated for complaints of abdominal pain and a painless umbilical nodule (Figure 1). 
On examination, a 3-cm exophytic necrotic lesion with mucoid and bloody discharge, ascites, and an abdominal mass growth were noted. A biopsy was performed and revealed a poorly differentiated carcinoma with extensive ulcerated necrotic areas infiltrating fibroadipose tissue.
As magnetic resonance was not available, computed tomography was performed and showed ascites, diffuse peritoneal carcinomatosis including an umbilical implant, multiple pulmonary nodules, bilateral pleural effusion, and retroperitoneal lymphadenopathy (Figures 2 and 3 ).

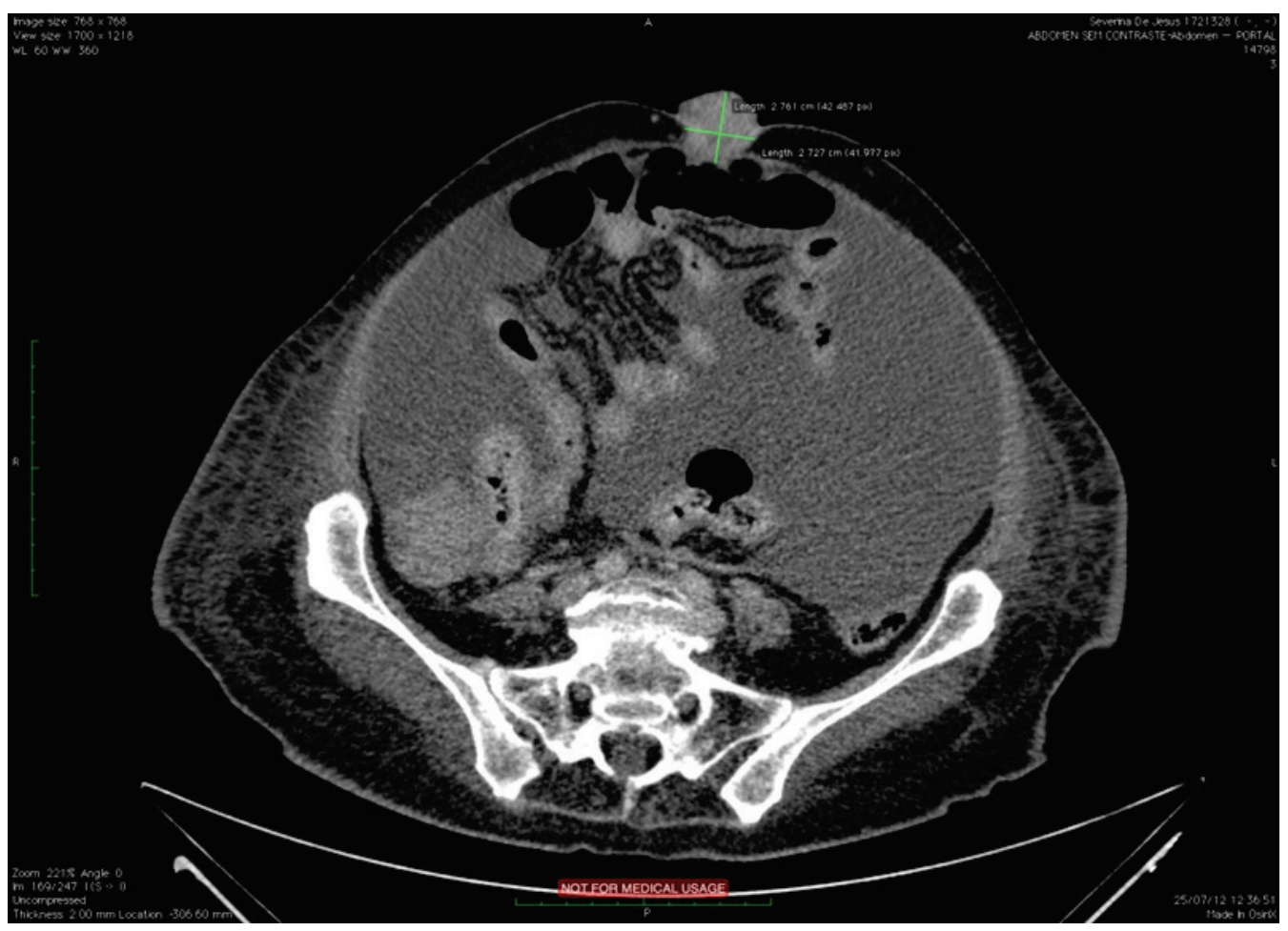

\section{Figure 2: Computed tomography showing umbilical nodule}

Paracentesis was performed, and hematic liquid had the following properties: mononuclear cells, 4.2 $\mathrm{g} / \mathrm{dL}$ total protein, a serum ascitesalbumin gradient of $<1.1 \mathrm{~g} / \mathrm{dL}$, and negative culture.

The treatment was palliative with local excision.

The patient developed respiratory distress and intestinal sub occlusion. Death occurred 63 days after SMJN diagnosis.

\section{Discussion}

SMJN is an umbilical tumor usually associated with advanced cancer. An umbilical mass can present as a benign lesion, but also as a primary or metastatic malignant tumor. ${ }^{1}$ Differential diagnoses include umbilical hernia, cutaneous endometriosis, pyogenic granuloma, melanocytic nevus, keloid, melanoma, squamous cell carcinoma, and basal cell carcinoma. $^{3}$

Umbilical nodules are due to a 
primary tumor and, despite being rare, they represent $38 \%$ of cases. Endometriosis is responsible for $32 \%$, and metastases, $30 \% .4$

SMJN is most commonly found in association with adenocarcinomas, and the most common primary site is the gastrointestinal tract (35$65 \%$ ). The gynecological tract is responsible for $12-35 \%$ of cases, and in these cases, the ovary is the most common primary site. ${ }^{3}$

SMJN may have different morphological characteristics, such as inflammatory erythematous thickening of the overlying skin, vascular appearance, fissures or ulceration, or features mimicking incarcerated umbilical hernia. ${ }^{3}$ In the present case, the nodule had a necrotic surface with bloody and mucoid discharge.

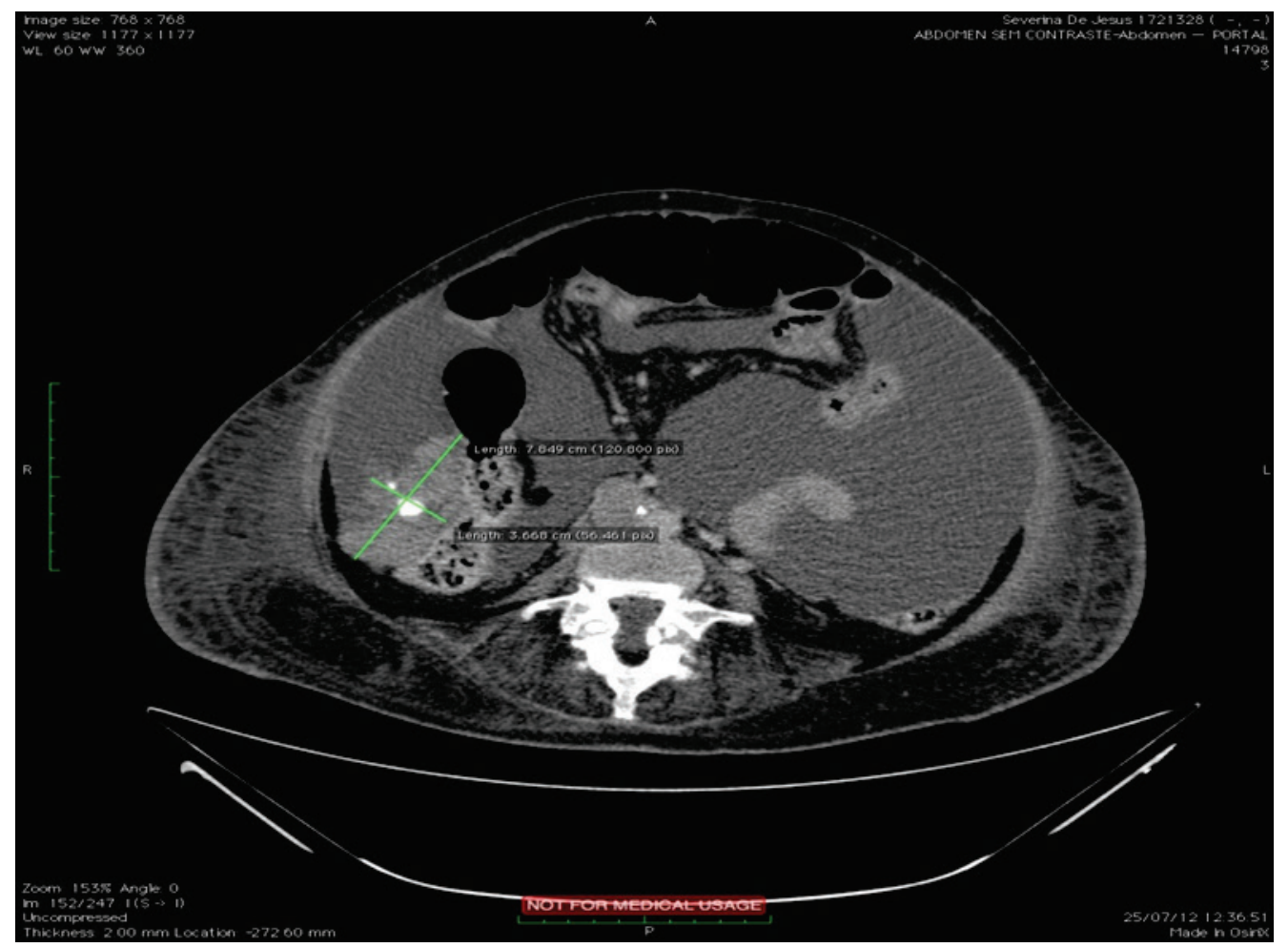

Figure 3: Computed tomography showing secondary peritoneal implantation in the right lower abdomen

Fagundes et al., in a retrospective analysis of 1211 patients with invasive cervical carcinoma, observed that clinical stage, endometrial extension prior to therapy, histology, and pelvic tumor control within each stage were indicators of distant dissemination. The frequency of metastases was greater when endometrial tumor extension was detected. ${ }^{5}$ Although endometrial extension is not included in the International Federation of Gynecology and Obstetrics staging, this extension is associated with a high risk of lymph node metastases and decreased disease-free and overall survival. ${ }^{6}$

The superficial squamous tumor replaces the entire endometrium in the intrauterine spread of cervical carcinoma. The tumor must satisfy 
three criteria (established by Fluhmann and modified by Kay) to be classified as primary endometrial carcinoma: 1) no coexistent endometrial adenocarcinoma, 2) no continuity between tumor and cervical squamous epithelium, and 3) no primary cervical carcinoma. ${ }^{7}$

Cervical carcinoma recurrence is most commonly loco-regional. ${ }^{8}$ Typical manifestations of recurrence involve the pelvis (preserved cervix, vagina or vaginal cuff, parametrium, bladder, rectum, ureters and ovaries) and lymph nodes. ${ }^{9}$ However, with the increasing use of pelvic irradiation, less typical patterns of recurrence such as peritoneal carcinomatosis can occur. ${ }^{10}$ These patterns are being recognized with greater frequency in patients with suspected recurrence because of the advancements in imaging studies.

Metastases from cervical carcinoma are usually predictable. Distant metastasis occurs commonly in the lung, liver, and bone. ${ }^{11}$ Umbilical metastasis is extremely rare, and the histological type most frequently found is adenocarcinoma. ${ }^{12}$

Our patient recurred with ascites, diffuse peritoneal carcinomatosis, umbilical nodule, bilateral pleural effusion, multiple pulmonary nodules, and retroperitoneal lymphadenopathy.

Skin metastasis is a late manifestation in cervical cancer patients and is an indication of uncontrolled or widespread metastasis. Most skin metastases occur after an interval of less than 21 months. The mean survival of patients with umbilical metastasis is 3 months. ${ }^{8}$
We presume that the progressive involvement of the peritoneum due to squamous cell carcinoma might have led to spread to the umbilicus, and this was likely responsible for the unusual course of this case.

At the time of umbilical metastasis diagnosis, the cancer was deemed incurable and the umbilical metastasis was an indication of recurrence. SMJN treatment remains palliative either by radiation, chemotherapy, surgery alone or in combinations. ${ }^{8}$

\section{Conclusion}

Sister Mary Joseph nodule is a sign of uncontrolled disease. Although similar cases have been reported in the literature, this manifestation of the squamous cell cervical cancer is rare and this clinical course associated with endometrial extension and peritoneal carcinomatosis is unique.

\section{References}

1. Larentzakis $A$, Theodorou $D$, Fili $K$, Manataki A, Bizimi V, Tibishrani M, Katsaragakis S. Sister Mary Joseph's nodule: Three case reports. Cases J. 2008 Sep 24;1(1):182. doi: 10.1186/17571626-1-182. PubMed PMID: 18816407

2. Palaniappan $M$, Jose $W M$, Mehta A, Kumar K, Pavithran K. Umbilical metastasis: a case series of four Sister Joseph nodules from four different visceral malignancies. Curr Oncol. 2010 Nov;17(6):78-81. http://dx.doi.org/10.3747/co.v17i6.6 84. PubMed PMID: 21151414. 
3. Al-Mashat F, Sibiany AM. Sister Mary Joseph's nodule of the umbilicus: is it always of gastric origin? A review of eight cases at different sites of origin. Indian $\mathrm{J}$ Cancer. 2010 Jan-Mar;47(1):65-9. doi: $\quad 10.4103 / 0019-509 \times .58862$. PubMed PMID: 20071793.

4. Gabriele $R$, Conte $M$, Egidi $F$, Borghese M. Umbilical metastases: current viewpoint. World J Surg Oncol. 2005 Feb 21;3(1):13. http://dx.doi.org/10.1186/1477-

7819-3-13. PubMed PMID: 15723695.

5. Fagundes $\mathrm{H}$, Perez CA, Grigsby PW, Lockett MA. Distant metastases after irradiation alone in carcinoma of the uterine cervix. Int $J$ Radiat Oncol Biol Phys. 1992;24(2):197-204. PubMed PMID: 1526855.

6. Hope AJ, Saha P, Grigsby PW. FDG-PET in carcinoma of the uterine cervix with endometrial extension. Cancer. 2006 Jan 1;106(1):196-200.

http://dx.doi.org/10.1002/cncr.2157 3. PubMed PMID: 16302228.

7. Marwah N, Garg M, Singh S, Sethi $D$, Sen R. Unusual form of squamous cell carcinoma of the cervix extending in situ into the endometrium: Three case reports and review of literature. Int $\mathrm{J} \mathrm{Appl}$ Basic Med Res. 2012 Jul;2(2):13941. doi: 10.4103/2229516X.106359. PubMed PMID: 23776829.
8. Behtash $\mathrm{N}$, Mehrdad $\mathrm{N}$, Shamshirsaz A, Hashemi R, Amouzegar Hashemi F. Umblical metastasis in cervical cancer. Arch Gynecol Obstet. 2008 Nov;278(5):489-91. doi: 10.1007/s00404-008-0617-4. Epub 2008 Apr 1. PubMed PMID: 18379810.

9. Fulcher AS, O'Sullivan SG, Segreti EM, Kavanagh BD. Recurrent cervical carcinoma: typical and atypical manifestations. Radiographics. 1999 Oct;19 Spec No:S103-16; quiz S264-5. PubMed PMID: 10517448.

10. Sala E, Wakely S, Senior E, Lomas D. MRI of malignant neoplasms of the uterine corpus and cervix. AJR Am J Roentgenol. 2007 Jun;188(6):1577-87. PubMed PMID: 17515380.

11. Elamurugan TP, Agrawal A, Dinesh $\mathrm{R}$, Aravind $\mathrm{R}$, Naskar $\mathrm{D}$, Kate $\mathrm{V}$, Reddy R, Elamurugan S; Siddaraju, Basu D; Parthasarathy. Palmar cutaneous metastasis from carcinoma cervix. Indian J Dermatol Venereol Leprol. 2011 Mar-Apr;77(2):252. doi: 10.4103/0378-6323.77486. PubMed PMID: 21393976.

12. Touraud JP, Lentz N, Dutronc $Y$, Mercier E, Sagot P, Lambert D. [Umbilical cutaneous metastasis (or Sister Mary Joseph's nodule) disclosing an ovarian adenocarcinoma]. Gynecol Obstet Fertil. 2000 Oct;28(10):719-21. French. PubMed PMID: 11244633. 